\title{
THE SURGICAL MANAGEMENT OF FALLOT'S TETRALOGY
}

\author{
D. N. Ross, B.Sc., M.B., F.R.C.S.
}

Consultant Thoracic Surgeon, Guy's Hospital, London, S.E.I

FaLlor's tetralogy is a condition of cyanotic congenital heart disease associated with pulmonary stenosis and a ventricular septal defect. The right ventricular hypertrophy and overriding aorta are probably a result of the altered hæmodynamics.

The essential features of the condition are cyanosis, pulmonary stenosis and a ventricular septal defect. Consequently, the so-called Trilogy of Fallot is excluded from consideration since these cases have no ventricular septal defect. Further, the term is misleading and should be dropped. Cases of ventricular septal defect with mild pulmonary stenosis and no cyanosis at rest or on exertion are also excluded from consideration under the definition presented above. Finally, the term Pentalogy of Fallot provides no useful information. This condition is better known as Fallot's tetralogy with an additional atrial septal defect.

\section{Hæmodynamics}

The ventricular septal defect in Fallot's tetralogy is invariably large so that there is a free communication between the right ventricle, the left ventricle, and the aorta. The obstruction to the outflow tract of the right ventricle is also severe with the result that when the right ventricular pressure rises in systole to systemic levels, the right ventricle discharges a large proportion of its contained desaturated blood into the left ventricle and aorta. Only a small proportion of the right ventricular ejection passes through the pulmonary stenosis to the lungs.

The clinical picture resulting is one of cyanosis as a result of unsaturated blood in the aorta. The hæmodynamic effect is that of equal systolic pressures in the right ventricle, left ventricle and aorta (Fig. I). The diminished blood flow to the lungs means that an inadequate volume of blood will be oxygenated there. This inadequate blood flow, and not the cyanosis, accounts for the patient's disability. Recognition of this fact was the basis of the original Taussig-Blalock operation designed to increase the blood flow to the lungs.

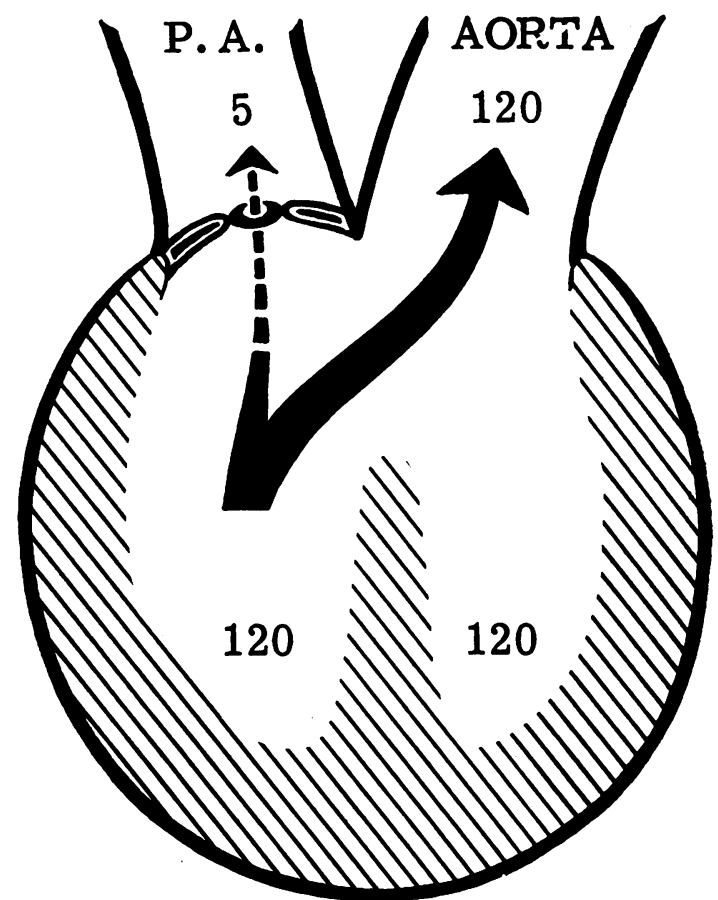

FIG. 1.-The relative volumes and direction of the right ventricular blood flow are shown together with the approximate pressures in the ventricles and great vessels.

\section{Anatomy}

The pulmonary stenosis may be purely valvar in site with the valve orifice a few millimetres in diameter and often lying eccentrically with poorlydefined valve commissures.

More commonly the obstruction is infundibular in type consisting of a localized hypertrophy of the muscle and a thickening of the overlying endocardium. This infundibular obstruction may lie in a high, medium or low position in relation to the pulmonary valve. Where it is low in position there is a well-formed and thin-walled infundibular chamber beyond it (Fig. 2). This is an example of an area of post-stenotic dilatation 


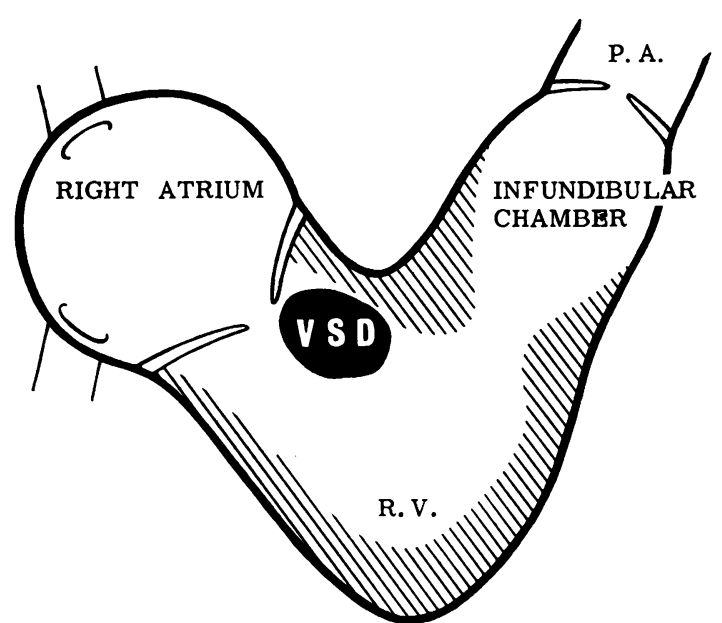

FIG. 2.-Diagrammatic section through the right heart showing the thin-walled infundibular chamber beyond the obstruction and the ventricular septal defect lying proximal.

occurring beyond an obstruction. Not infrequently an infundibular and valvar stenosis coexist.

The ventricular septal defect in Fallot lies beneath the supraventricular crest or proximal to the infundibular obstruction. It is therefore within the high-pressure zone of the right ventricle. In size it usually approximates the diameter of the aorta and it is often partly overlapped by the septal cusp of the tricuspid valve. The bundle of $\mathrm{His}$ runs along the postero-inferior margin of the defect (Fig. 3). Since the advent of open-heart surgery these aspects of the surgical anatomy have acquired considerable importance.

Externally the ventricles are covered by numerous distended and tortuous coronary vessels well in excess of the normal coronary vascular pattern (this is thought to be the result of anoxia).

The pulmonary artery is usually small and of low tension, while the aorta often has a diameter two to three times as great and appears to arise partly from the right ventricle. The relative size of the pulmonary artery and aorta reflect the degree of shunting of right ventricular blood into the aorta at the expense of the pulmonary artery and is consequently a guide to the severity of the condition.

\section{Clinical Features}

Cyanosis. Children with Fallot's tetralogy are cyanosed from birth or the cyanosis is noticed within the first few weeks. The degree of cyanosis varies and is increased by crying and exertion.

In severe cases the child is subject to "cyanotic attacks'. These are characterized by attacks of deep cyanosis and may be accompanied by loss of

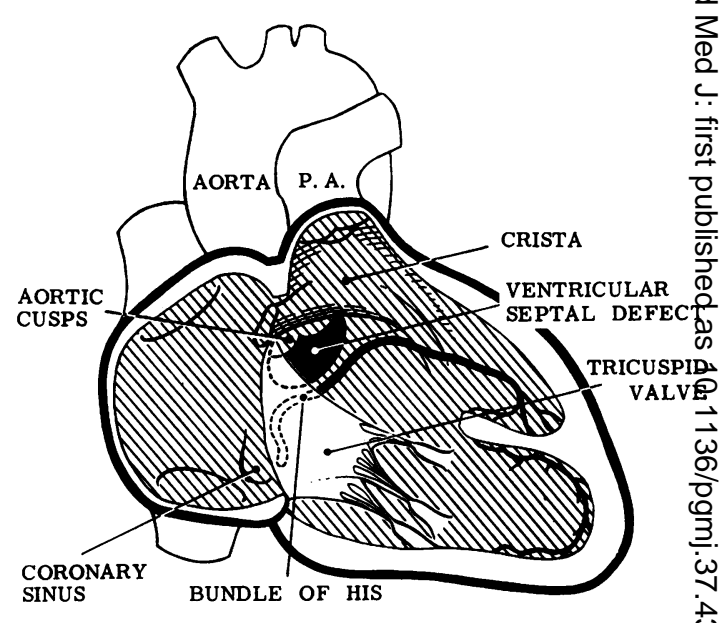

FIG. 3.-Surgical anatomy of the ventricular septa defect in Fallot showing relationships of bundle $0 \delta$ His, aortic cusps, crista and septal cusp of tricusp valve.

consciousness or death. These attacks may be precipitated by a 'spasm' or increased tonus of the infundibulum. This will have the effect of directing all or most of the right ventricular blgoe into the aorta with the production of cerelot anoxia.

Alternatively, the cyanotic attacks may be cipitated by diminution of the peripheral systemio resistance which will have the same effect of directing blood preferentially into the aorta at the expense of the pulmonary artery.

Squatting. Cases of Fallot's tetralogy adopt $\frac{\mathbb{Q}}{q}$ characteristic squatting attitude when they ar $\overrightarrow{\mathcal{E}}$ tired and some children even sit in this position in bed. The effect of the acute flexion at kne and thigh may be to trap blood in the legs an so reduce the load of returning venous blood ta the heart. On the other hand, by increasing the. systemic vascular resistance (aided by splanchnic bed compression by the thighs) the effect may be to divert more blood from the aorta to the lungso

It is rare for a case of Fallot's tetralogy not to give a history of squatting, and where this absent one should be wary of the diagnosis. $\frac{7}{0}$

Dyspncea. All of these cases are dyspnœic to greater or lesser degree, depending largely on the adequacy of the blood flow to the lungs.

Clubbing. Finger clubbing is a characteristi feature of the condition; the degree of clubbing is usually proportional to the severity of the cyanosis. The toes also show clubbing and severely cyanotic cases the tip of the nose als may be clubbed.

Cardiovascular System. Examination reveals normal pulse volume, distended veins and ${ }_{\oplus}$ warm periphery. The right ventricular activit 
is moderately increased on palpation and a systolic thrill is often palpable in the second and third left intercostal spaces. A pulmonary systolic murmur is characteristically heard and is followed by the loud banging single sound of aortic valve closure. Where the pulmonary stenosis is severe the thrill is absent and the systolic murmur may be insignificant.

\section{Special Investigations}

Polycythamia. This again will vary with the degree of cyanosis and is a good guide to the severity of the condition pre-operatively. Postoperatively it offers an objective assessment of the degree of improvement.

Electrocardiogram. This is not particularly characteristic but usually shows right ventricula $r$ hypertrophy of a moderate degree. Marked right ventricular hypertrophy is unusual and raises the possibility of an intact ventricular septum.

Chest Radiograph. This is useful. The lung fields are typically oligæmic and the pulmonary vasculature is often spidery.

The heart contour may be sabot-shaped in severe cases, but this picture is by no means invariable. The aorta is usually large and is rightsided in about $25 \%$ of cases.

Angiocardiography. This is important in the surgical assessment of the case. The points to establish are:

(a) Confirmation of the diagnosis.

(b) Precise anatomy of the right ventricle outflow obstruction.

(c) Size of the pulmonary artery branches and the aorto/pulmonary artery ratio.

(a) The contrast medium passes from right atrium to right ventricle and from this chamber fills the pulmonary artery and aorta simultaneously. This confirms the presence of pulmonary stenosis, a ventricular septal defect, and a right-to-left shunt.

(b) The anatomy of the infundibular and pulmonary valve obstructions can be identified. These features are seen most clearly in the lateral view (Fig. 4).

(c) The ratio of the size of the pulmonary artery to the aorta can be measured. Where the diameter of the pulmonary artery is over $50 \%$ of that of the aorta, it suggests that this vessel will be of adequate size to deal with the output of blood from the right ventricle once the ventricular septal defect has been closed. It is important to estimate the diameter of the pulmonary artery at the position of the valve ring (Fig. 5), since this is likely to be the narrowest area and cannot easily be enlarged without causing pulmonary valve regurgitation.

Selective right ventricular angiography, achieved

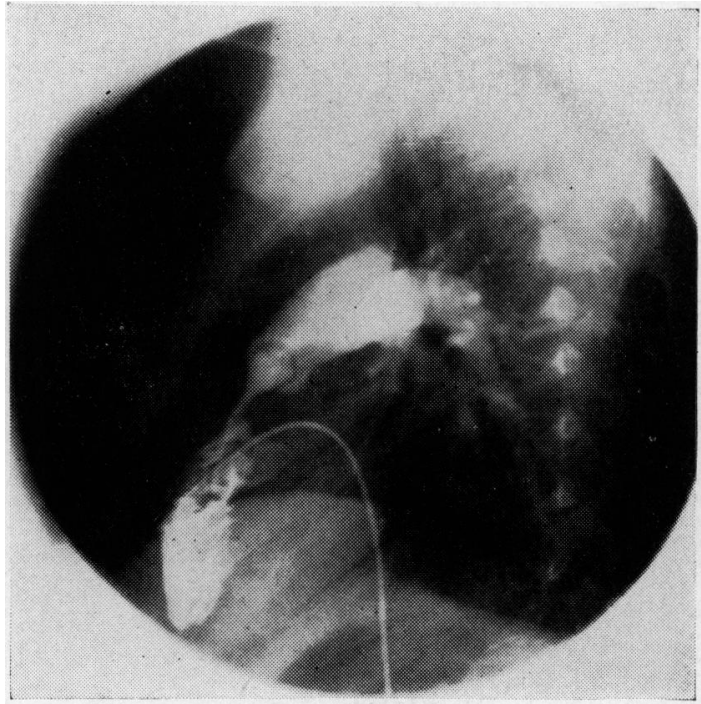

a.

b.

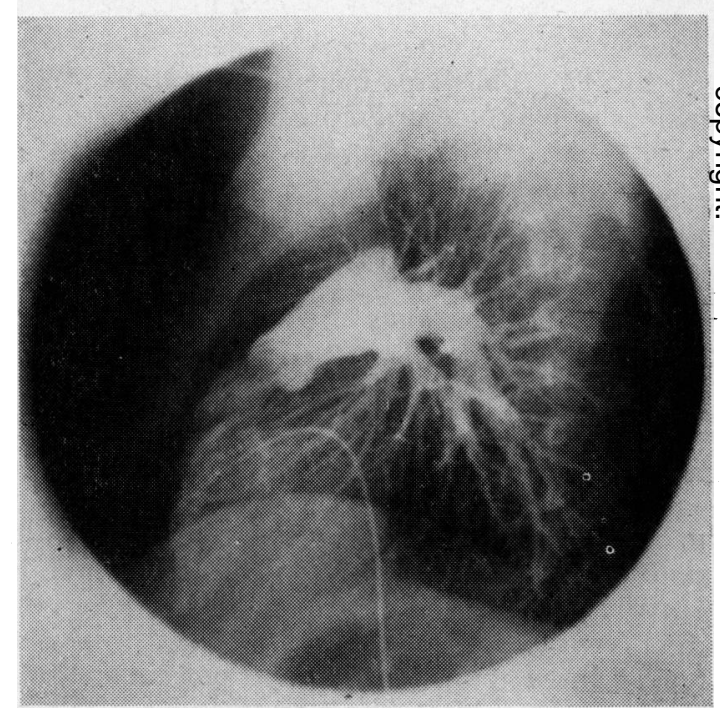

FIG. 4, $a$ and $b$.- - Lateral angiocardiogram showing the anatomy of the right ventricular outflow in systole and diastole. There is a valve stenosis present.

by means of a wide-bore catheter into the right ventricle, gives excellent anatomical detail.

Asymmetry of the right and left main pulmonary arteries and their peripheral distribution is common in Fallot's tetralogy.

Cardiac Catheterization. This examination is not often necessary, but where there is difficulty in differentiating Fallot from other conditions it 


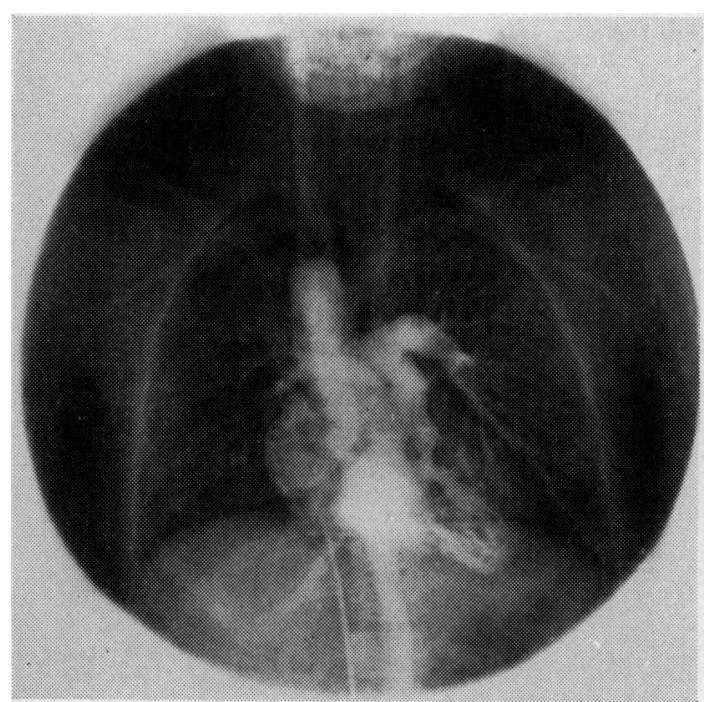

Fig. 5.-Angiocardiogram shows simultaneous filling of pulmonary artery and aorta. The pulmonary artery diameter suggests the case is suitable for a one-stage corrective operation.

is valuable. This is particularly true if combined with selective angiocardiography.

The catheter demonstrates the presence of a pulmonary stenosis and also the fact that the right and left ventricular pressures are typically equal.

\section{Differential Diagnosis}

Other cyanotic congenital heart disorders should be excluded. Most important is the condition of pulmonary stenosis with a closed ventricular septum and reverse shunt through an atrial septal defect. Since there is no ventricular septal defect to act as a safety valve, the clue to the diagnosis rests on the recognition of an obstructed right ventricle. Disability is likely to be severe, the right ventricle load is usually reflected in distended neck veins with giant pulsations as a result of increased atrial contractions. There may, in addition, be enlargement of the liver. Also the right ventricular action is powerful to palpation and there will be a long systolic murmur and quiet second heart sound. The electrocardiograph will confirm the presence of right ventricular hypertrophy usually of a degree in excess of that found in Fallot's tetralogy. The radiographic contour of the heart is more globular than in Fallot's tetralogy and there is usually a distinct post-stenotic dilatation of the pulmonary artery.

Rarer conditions to be considered are transposition of the great vessels, total anomalous pulmonary venous drainage, and some cases of $\frac{\varrho}{2}$ truncus arteriosus. The diagnosis of these con- $\frac{\mathbb{D}}{\mathrm{D}}$ ditions rests on clinical examination and con- $\frac{2}{c}$ firmation by special investigational techniques.

\section{Surgical Principles}

Surgical treatment aims at relieving the disability and at restoring the normal anatomy $\frac{\infty}{7}$ wherever possible. The main disability stems $\stackrel{\mathbb{Q}}{\Omega}$ from the pulmonary stenosis. The ventricular septal defect, however, represents an additional hæmodynamic burden to the heart.

There can be no doubt that the ideal treatment is to remove the pulmonary stenosis and to close the ventricular septal defect provided this can be achieved with an acceptable surgical risk. Where this total correction is not possible, relief of the pulmonary stenosis should be the primary sur- is gical consideration.

\section{Surgical Management}

The Blalock-Taussig operation aims at overcoming the pulmonary stenosis and poor pulmonary flow by circumventing the obstruction. The patient's disability is dramatically improved and the relief afforded by the operation provides striking confirmation of the view that the dis $\bar{\Phi}$ ability in Fallot stems from the pulmonare stenosis. Its disadvantage lies in the fact that i does nothing to relieve the burden of the righis ventricle and the presence of the anastomosis adds an additional problem when the case is subsequently dealt with by total corrective surgery.

On the other hand, the early results are excellent and the increased blood flow to the lungs creates conditions for the development of the pulmonary vasculature and left heart chambers. These latter features may be important in ensuring the success of the subsequent corrective surgery.

The Brock operation for Fallot's tetralogy represents a direct but closed operation on the pulmonary stenosis. Where the obstruction is at valve level, pulmonary valvotomy is performed and in cases of infundibular obstruction the infundibular muscle is resected piecemeal with the aid of Brock's infundibular punches. The effect of the Brock operation is to allow the development not only of the pulmonary vascular bed and left heart but also of the right ventricular outflow tract under the influence of the increased blood flow.

Both the Blalock and Brock operations leave the patient with a ventricular septal defect and it is likely that these operations will in future be restricted to very young or high-risk cases as a preliminary or first-stage procedure. The closure of the ventricular septal defect and removal of the 
residual pulmonary stenosis can then be achieved at a subsequent operation.

Correction of the pulmonary stenosis and closure of the ventricular septal defect as a onestage procedure represents the ideal treatment for Fallot's tetralogy. It should be the main aim of surgical management. In order to ensure success, an efficient heart-lung apparatus and organized team must be available. The machine must be able to maintain the circulation for a minimum of 60 minutes and there must be skilled post-operative nursing care.

In assessing a case for total correction, the size of the right ventricular outflow and pulmonary artery is of importance since the whole cardiac output must be accommodated through this channel after closure of the ventricular septal defect. Where the diameter of the pulmonary artery is grossly inadequate, many surgeons prefer a preliminary Brock or Blalock operation.

With regard to the technical aspect of the extracorporeal circulation, in general, the flow requirements are proportionately higher in Fallot's tetralogy than in other cases of open-heart surgery. This results from the often considerable bronchial arterial 'leak' of blood from the systemic circuit to the lungs (Fig. 6). Consequently the volume of oxygenated blood available for perfusion of the systemic circuit may be inadequate for the physiological needs of the brain and other organs. A further disadvantage of this 'leak' is the embarrassing volume of bronchial blood returning to the operative field via the pulmonary veins and left atrium. As much as one-quarter to one-third of the pump output may be dissipated in this manner.

The capacity of the pump-oxygenator must therefore be able to meet these increased flow requirements and this applies particularly to adult cases. In many centres, including Guy's Hospital, this difficulty is overcome by using moderate hypothermia to a temperature of about $30^{\circ} \mathrm{C}$. This ensures adequate reserves of oxygenating capacity.

It is a further advantage to use hypothermic arrest of the heart's action in order to secure ideal operating conditions in a quiescent heart. This is most simply achieved by packing ice-saline crystals around the heart as suggested by Hufnagel (Ross, 196r) (Fig. 7). Under these circumstances the increased bronchial return is also more easily dealt with than in the beating heart, and in fact the conditions approximate those available under profound hypothermia and circulatory arrest.

The right ventricle is opened through a reasonably short vertical incision over the outflow tract, taking care to avoid the large coronary arteries. Exceptionally, the main coronary blood supply

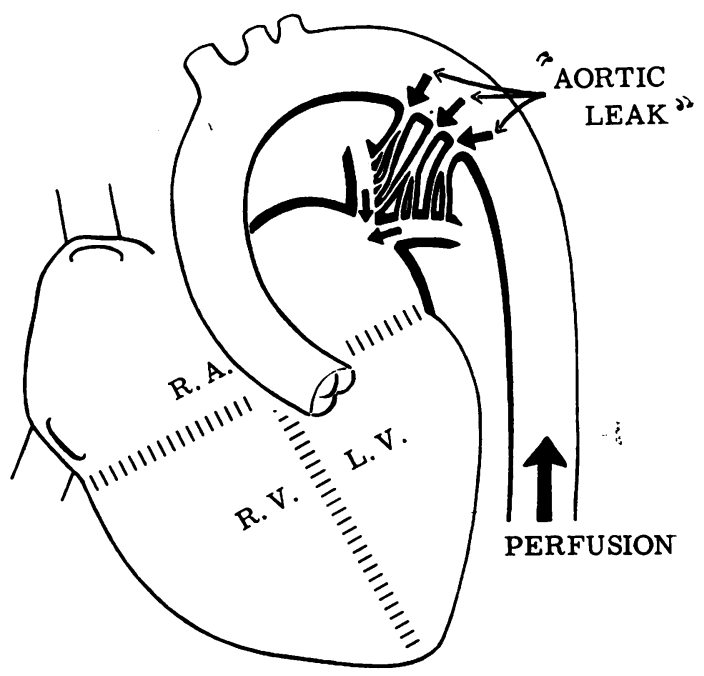

FIG. 6.-This diagram indicates the bronchial "leak" of blood into the pulmonary veins and left atrium.

to the left ventricle may course across the line of incision so that the operation may have to be abandoned where the incision cannot be planned to avoid this vessel. Some surgeons have been able to dissect the coronary out of the myocardium and retract it out of the line of the incision.

The crista supraventricularis is a well-developed muscular ridge in Fallot's tetralogy with thickened overlying endocardial 'icing'. In addition, the trabeculæ carneæ are usually seen as grossly hypertrophied muscular bundles. There may be an associated valve stenosis and this should be looked for and dealt with before becoming preoccupied with the infundibulum or ventricular septal defect.

The crista is resected generously by sharp dissection, taking care not to encroach upon the aortic valve ring and cusps which should be identified adjacent to its deep surface. Attention is then directed to the trabeculæ carneæ. These are divided extensively rather than excised in order to increase the mobility and diameter of the right ventricular outflow tract.

The ventricular septal defect is then closed, either by a direct suture technique (Kirklin, Ellis, McGoon, DuShane and Swan, 1959) or with the aid of a plastic or pericardial prosthesis. In all cases the defect is large in relation to the size of the heart and has a fleshy muscular edge as opposed to the tougher and more tendinous edge in cases of isolated ventricular septal defect.

Once the ventricular septal defect is closed it is important to maintain decompression of the left heart chambers and pulmonary bed, either by 


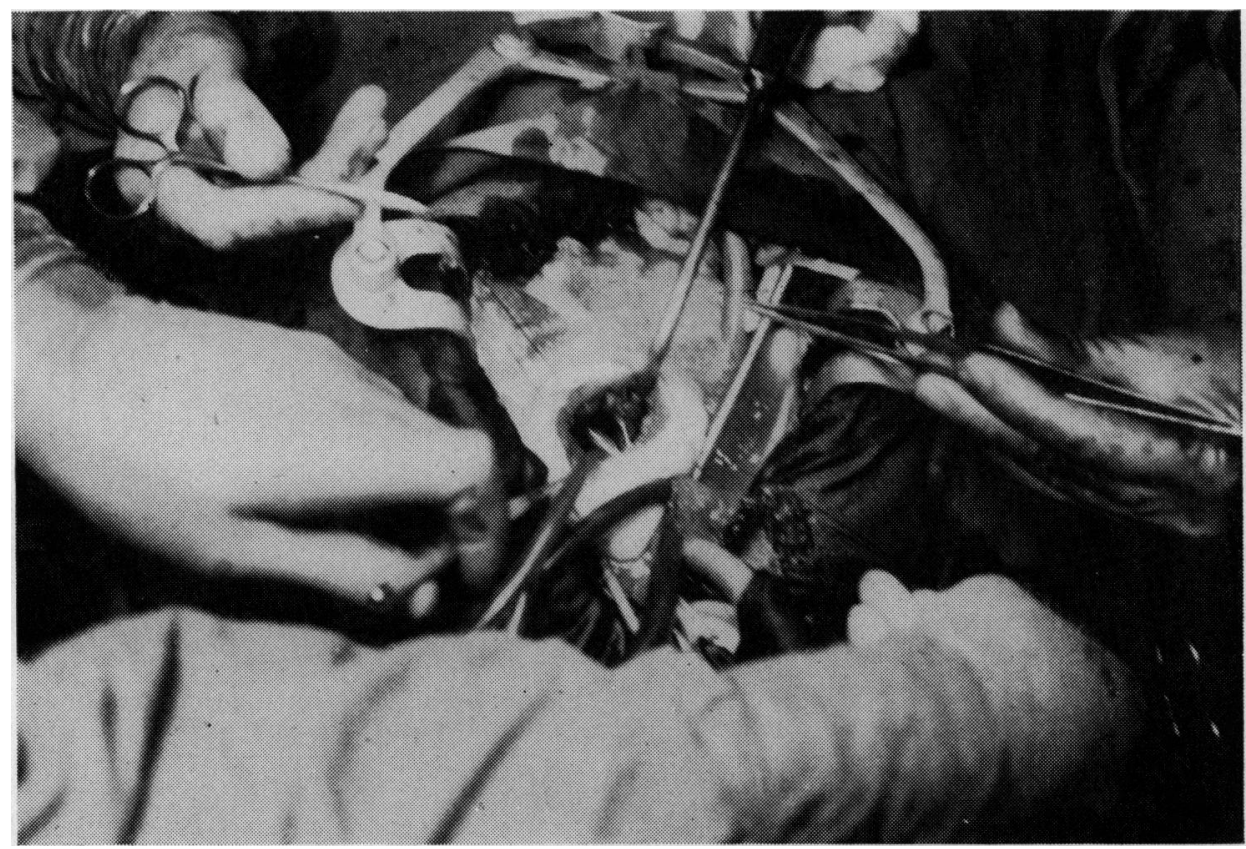

FIG. 7.-Dry operating conditions within the ice-arrested heart in Fallot's tetralogy.

venting these through a sucker in the pulmonary artery or by means of a previously placed left atrial sucker. The heart action can be restarted at this stage so that one can confirm the closure of the ventricular septal defect.

In a correctly-selected case it is now possible to close the right ventriculotomy by direct suture and this is the practice at Guy's Hospital. Where total correction is attempted in cases with a poorly-developed right ventricular outflow tract or pulmonary artery, it is the custom to insert an elliptical gusset of plastic or pericardium at this stage in order to increase the diameter of the right ventricular outflow. The gusset may even be carried through the pulmonary valve ring into the pulmonary artery bifurcation in the most severe cases (Fig. 8), but this has the effect of inflicting severe pulmonary regurgitation which may be a later source of disability.

Once heart action is restored, pressures are recorded in the right and left ventricles and pulmonary artery. Under favourable conditions the right ventricular pressure should be less than half of that in the left ventricle and the pressure gradient between the right ventricle and pulmonary artery should be absent or small. A considerable residual gradient represents a serious post-operative load to the right ventricle which has the additional burden of an incision.
Post-operative bleeding following by-pass often a hazard, particularly in polycythæmic caseso and no certain method of dealing with this coms plication has been offered. Blood loss is measure carefully and is replaced with fresh blood unt bleeding stops. This increased bleeding tendence in severe Fallot's tetralogy appears to be one $0 \vec{\Phi}$ the outstanding problems in the surgery of this condition.

As in the closure of any ventricular septal defect, heart block is a danger to be avoided at al costs since permanently-established block has $\frac{3}{3}$. poor immediate and late prognosis. Heart block when it occurs, calls for the use of the electricad pace-maker and heart action must be carefully monitored on the electrocardiograph.

Post-operatively cardiac output may be inade quate during the first month to six weeks, and is usual to keep the patient in hospital at least month post-operatively. This deficient cardiae output may be further evidence of the difficulties of the left ventricle in dealing with its neet work load.

\section{Summary}

At present the surgical management of Fallot $\stackrel{0}{\stackrel{s}{s}}$ tetralogy is reasonably standardized. Most case 8 are satisfactorily dealt with by a one-stage tota 


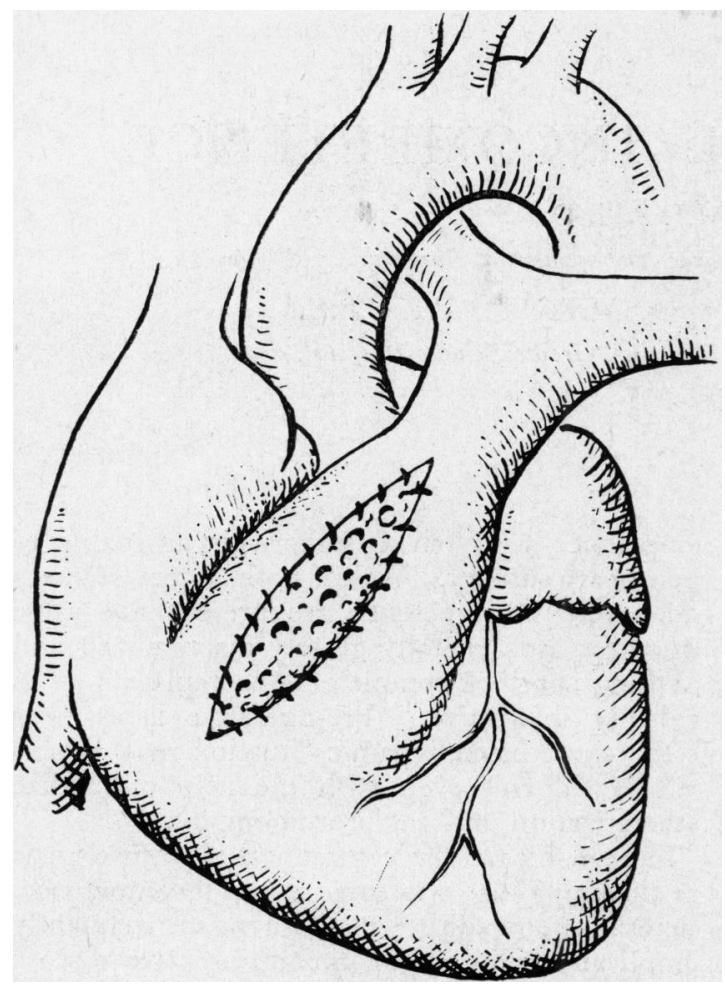

corrective procedure. This should be achieved with a 15 to $20 \%$ mortality.

In very young patients (usually under four) requiring surgical relief and in severely incapacitated cases at any age, a preliminary Blalock or Brock operation affords considerable clinical improvement. A preliminary operation has the effect of developing the right ventricular outflow, the pulmonary vascular bed and the left atrium and ventricle. These factors, together with the reduced polycythæmia and bleeding tendency, result in an improvement of the clinical condition and present a less formidable operative risk for subsequent corrective surgery.

Fig. 8.-Diagram of a plastic gusset in the right ventricular outflow tract.

\section{REFERENCES}

Kirklin, J. W., Ellis, F. H., McGoon, D. C., DuShane, J. W., and Swan, H. J. C. (1959): Surgical Treatment for the Tetralogy of Fallot by Open Intracardiac Repair, f. thorac. Surg., 37, 22.

Ross, D. N. (196r): Ice Arrest of the Heart, Lancet, ii, 293. 